70th International Astronautical Congress, Washington D.C. Copyright @2019 by Mauro Pontani and Fabio Celani.

Published by the IAF, with permission and released to the IAF to publish in all forms

IAC-19-C1.8.8

\title{
VARIABLE-TIME-DOMAIN NEIGHBORING OPTIMAL GUIDANCE AND ATTITUDE CONTROL OF LOW-THRUST LUNAR ORBIT TRANSFERS
}

\author{
Mauro Pontani \\ Department of Astronautical, Electrical, and Energy Engineering, Sapienza University of Rome, Italy \\ mauro.pontani@uniroma1.it \\ Fabio Celani \\ School of Aerospace Engineering, Sapienza University of Rome, Italy \\ fabio.celani@uniroma1.it
}

Lunar orbit dynamics and transfers at low altitudes are subject to considerable perturbations related to the gravitational harmonics associated with the irregular lunar mass distribution. This research proposes the original combination of two techniques applied to low-thrust lunar orbit transfers, i.e. (i) the variable-time-domain neighboring optimal guidance (VTD-NOG), and (ii) a proportional-derivative attitude control algorithm based on rotation matrices (PD-RM). VTD-NOG belongs to the class of feedback implicit guidance approaches, aimed at maintaining the spacecraft sufficiently close to the reference trajectory. This is an optimal path that satisfies the second-order sufficient conditions for optimality. A fundamental original feature of VTD-NOG is the use of a normalized time scale, with the favorable consequence that the gain matrices remain finite for the entire time of flight. VTD-NOG identifies the trajectory corrections by assuming the thrust direction as the control input. Because the thrust direction is fixed with respect to the spacecraft, VTD-NOG generates the desired orientation pursued by the attitude control system. A proportional-derivative approach using rotation matrices (PD-RM) is employed in order to drive the actual spacecraft orientation toward the desired one. Reaction wheels are considered as the actuators that perform attitude control. Extensive Monte Carlo simulations are performed, in the presence of nonnominal flight conditions related to (i) lunar gravitational harmonics, (ii) gravitational pull of the Earth and the Sun as third bodies, (iii) unpredictable propulsive fluctuations, and (iv) errors on initial attitude. The numerical results unequivocally demonstrate that the joint use of VTD-NOG and PD-RM control represents an accurate and effective methodology for guidance and control of low-thrust lunar orbit transfers.

\section{INTRODUCTION}

Recently, several countries have shown an increasing interest toward robotic or human missions to the Moon. Most lunar mission architectures include a spacecraft that orbits the Moon at low altitude. Orbit transfers between different low-altitude lunar orbits often represent a crucial phase, which can be completed only if the space vehicle is equipped with an efficient and robust guidance and control system.

In this context, low-thrust propulsion may represent a valuable option. In fact, low-thrust propulsive systems can be proven to outperform high-thrust engines in terms of propellant budget, in a wide variety of practical scenarios, at the price of increasing considerably the time of fight. Propellant minimization represents the main purpose of low-thrust orbit transfer optimization, with the final aim of identifying the nominal path associated with the mission specifications. A variety of analytical and numerical techniques ${ }^{1-5}$ have been employed in the past for spacecraft trajectory optimization. However, in concrete scenarios, deviations from the nominal trajectory related either to the imperfect modeling of the space vehicle or to unpredictable environmental conditions affect the actual spacecraft dynamics. Driving a spacecraft along a specified path thus requires defining the corrective actions aimed at compensating the nonnominal behavior due to these deviations, while minimizing the additional fuel required to perform these corrective maneuvers.

The present research is intended to describe and apply an implicit guidance and control approach, capable of generating perturbed paths sufficiently close to the nominal trajectory, which is assumed to be optimal.

As a preliminary step, the general optimization methodology termed indirect heuristic method (IHM) ${ }^{6,7}$ is employed, with the intent of obtaining the nominal (optimal) path. The method at hand is based upon the joint use of the necessary conditions for optimality and a heuristic technique (e.g., the particle swarm algorithm). The subsequent, closely related problem is in driving the space vehicle along the optimal path. This requires defining the corrective actions aimed at compensating the nonnominal behavior of the space vehicle. This means that a feedback control law, or, equivalently, a closed-loop guidance algorithm, is to be 
defined, based on the current state, evaluated at prescribed sampling times. Neighboring Optimal Guidance (NOG) is an implicit guidance concept that relies on the analytical second order optimality conditions, in order to find the corrective control actions in the proximity of the reference path. In general, the neighboring optimal path originates from a perturbed state and is associated with the minimization of the second differential of the objective function. Several time-varying gain matrices, referring to the nominal trajectory, are defined, computed offline, and stored in the onboard computer. Only a limited number of works have been devoted to studying neighboring optimal guidance ${ }^{8-12}$. A common difficulty encountered in implementing the NOG consists in the fact that the gain matrices become singular while approaching the final time. As a result, the real-time correction of the time of flight can lead to numerical difficulties so relevant to cause the failure of the guidance algorithm.

This research is focused on the original combination of two techniques applied to low-thrust orbit transfers, i.e. (i) the recently-introduced variable-time-domain neighboring optimal guidance (VTD-NOG) ${ }^{13-15}$, and (ii) a proportional-derivative approach based on rotation matrices (PD-RM) for the attitude control algorithm. VTD-NOG belongs to the class of feedback implicit guidance approaches, aimed at finding the corrective control actions capable of maintaining the spacecraft sufficiently close to the reference trajectory. This is an optimal path that satisfies the second-order sufficient conditions for optimality. A fundamental original feature of VTD-NOG is the use of a normalized time scale as the domain in which the nominal trajectory and the related vectors and matrices are defined. VTD-NOG identifies the trajectory corrections by assuming the thrust direction as the control input. Because the thrust direction is fixed with respect to the spacecraft, VTDNOG iteratively generates the desired attitude, which can be possibly discontinuous across subsequent guidance intervals. This circumstance implies that the actual orientation, which is subject to the spacecraft attitude dynamics, does not coincide with the desired orientation. Hence, the attitude control system must be capable of maintaining the actual spacecraft orientation sufficiently close to the desired one. Reaction wheels are considered as the actuators that perform attitude control. This technological solution is often employed onboard satellites with low-thrust propulsion ${ }^{16,17}$. The control law being adopted is proportional-derivativelike and uses directly the rotation matrices (PD-RM), because large attitude maneuvers may be required. Combinations of VTD-NOG and different types of PD attitude control have been employed for a lunar ascent problem $^{18}$, as well as for high-thrust and low-thrust LEO to GEO transfers ${ }^{19,20}$.
This study describes the application of VTD-NOG \& PD-RM to the low-thrust orbit transfer that starts from a low Moon orbit (LMO) and ends at injection into a coplanar LMO at greater altitude. As a first step, the optimal two-dimensional trajectory is derived, and represents the nominal path. Then, several deviations from nominal flight conditions are assumed, i.e. (i) gravitational perturbations related to the harmonics of the lunar gravitational field, (ii) third body attraction due to Sun and Earth, (iii) unpredictable oscillations of the propulsive thrust, and (iv) errors on initial attitude. Monte Carlo campaigns are performed, with the final aim of proving that the unified architecture based on the joint use of VTD-NOG and PD-RM indeed represents an effective guidance and control approach, capable of determining precise and fuel-efficient low-thrust orbit transfers, in the presence of nonnominal flight conditions.

\section{ORBIT TRANSFER OPTIMIZATION}

This paper addresses the problem of driving a spacecraft from an equatorial circular low Moon orbit (LMO) at altitude of $300 \mathrm{~km}$ to a final, coplanar LMO at altitude of $400 \mathrm{~km}$, in the presence of nonnominal flight conditions. Both trajectory and attitude dynamics of the space vehicle are modeled. This section is specifically devoted to defining the nominal transfer path. In this context, the space vehicle is modeled as a point mass. Subsequently, attitude dynamics is considered, with the final intent of determining the appropriate attitude control action.

Continuous low-thrust propulsion is employed to perform the transfer at hand. Let $c$ and $n_{0}$ denote the effective exhaust velocity of the propulsive system and the initial thrust acceleration. The thrust magnitude is assumed constant, thus the thrust acceleration $(T / \tilde{m})$ is

$$
\frac{T}{\tilde{m}}=\frac{n_{0} c}{c-n_{0} t}
$$

where $c$ is the (constant) effective exhaust velocity of the propulsive system, $n_{0}$ is the initial thrust acceleration (at $t_{0}$, set to 0 ), and $t$ is the actual time. The following nominal values are assumed: $n_{0}=0.0001 g_{0}$ and $c=30 \mathrm{~km} / \mathrm{sec}\left(g_{0}=9.8 \mathrm{~m} / \mathrm{sec}^{2}\right)$.

\section{II.I Formulation of the problem}

The spacecraft motion can be described in a convenient Moon-centered inertial reference frame (MCI), associated with the right-handed sequence of unit vectors $\left(\hat{c}_{1}, \hat{c}_{2}, \hat{c}_{3}\right)$, where $\left(\hat{c}_{1}, \hat{c}_{2}\right)$ lie in the Moon's equatorial plane, $\hat{c}_{3}$ points toward its rotation axis, and $\hat{c}_{1}$ is associated with the initial spacecraft position. The time-varying position can be identified by the following 
three variables: radius $r$, absolute longitude $\xi$, and latitude $\phi$, portrayed in Fig. I(a). The spacecraft velocity can be projected into the rotating frame $(\hat{r}, \hat{t}, \hat{n})$, where $\hat{r}$ is aligned with the position vector $\boldsymbol{r}$ and $\hat{t}$ is parallel to the $\left(\hat{c}_{1}, \hat{c}_{2}\right)$-plane (and in the direction of the spacecraft motion, cf. Fig. I(a)). The related components are denoted with $\left(v_{r}, v_{t}, v_{n}\right)$ and termed respectively radial, transverse, and normal velocity component. The state vector $\boldsymbol{x}$ (with components denoted with $\left.x_{k}(k=1, \ldots, 6)\right)$ of the spacecraft includes the variables associated with the position and velocity vectors and is given by

$$
\boldsymbol{x}:=\left[\begin{array}{llllll}
r & \xi & \phi & v_{r} & v_{t} & v_{n}
\end{array}\right]^{T}
$$

The spacecraft is controlled through the thrust direction, defined by the in-plane angle $\alpha$ and the out-of-plane angle $\beta$, both illustrated in Fig. I(b) (in which $\hat{T}$ is aligned with the thrust direction). Thus, the control vector $\boldsymbol{u}$ is

$$
\boldsymbol{u}:=\left[\begin{array}{ll}
u_{1} & u_{2}
\end{array}\right]^{T}=\left[\begin{array}{ll}
\alpha & \beta
\end{array}\right]^{T}
$$

The equations of motion, also termed state equations hence forward, govern the spacecraft dynamics, and involve the state vector $\boldsymbol{x}$ and the control vector $\boldsymbol{u}$,

$$
\begin{gathered}
\dot{r}=v_{r} \\
\dot{\xi}=\frac{v_{t}}{r \cos \phi} \\
\dot{\phi}=\frac{v_{n}}{r} \\
\dot{v}_{r}=-\frac{\mu}{r^{2}}+\frac{v_{t}^{2}+v_{n}^{2}}{r}+\frac{T}{\tilde{m}} \sin \alpha \cos \beta+a_{r} \\
\dot{v}_{t}=\frac{v_{t}}{r}\left(v_{n} \tan \phi-v_{r}\right)+\frac{T}{\tilde{m}} \cos \alpha \cos \beta+a_{t} \\
\dot{v}_{n}=-\frac{v_{t}^{2}}{r} \tan \phi-\frac{v_{r} v_{n}}{r}+\frac{T}{\tilde{m}} \sin \beta+a_{n}
\end{gathered}
$$

where $(T / \tilde{m})$ is given by Eq. [1] and $\mu\left(=4902.9 \mathrm{~km}^{3} / \mathrm{sec}^{2}\right)$ is the Moon gravitational parameter. The symbols $a_{r}, a_{t}$, and $a_{n}$ denote the overall perturbing acceleration components, related to third bodies and higher harmonics of the lunar gravitational fields. In general, these components have modest magnitude and depend on the spacecraft position and velocity in a rather complicated way. Therefore, they are neglected for trajectory optimization, whereas they are being taken into account later in this work, while testing the guidance and control algorithm. Hence, Eqs. [4]-[9] can be written in the general compact form

$$
\dot{\boldsymbol{x}}=\tilde{\boldsymbol{f}}(\boldsymbol{x}, \boldsymbol{u}, t)
$$

Due to the definition of the inertial frame in relation to the initial spacecraft position, the initial conditions (denoted with the subscript " 0 ") are

$$
\begin{array}{lll}
r_{0}=R_{i} & \xi_{0}=0 & \phi_{0}=0 \\
v_{r 0}=0 & v_{t 0}=\sqrt{\frac{\mu}{R_{i}}} & v_{n 0}=0
\end{array}
$$

where $R_{i}$ is the radius of the initial lunar orbit. The final conditions (denoted with subscript " $f$ ") are

$r_{f}=R_{f} \quad \phi_{f}=0 \quad v_{r f}=0 \quad v_{t f}=\sqrt{\frac{\mu}{R_{f}}} \quad v_{n f}=0$

where $R_{f}$ is the radius of the final lunar orbit. Equations [11]-[12] can be written in compact form as

$$
\boldsymbol{\psi}\left(\boldsymbol{x}_{0}, \boldsymbol{x}_{f}, t_{f}\right)=\mathbf{0}
$$

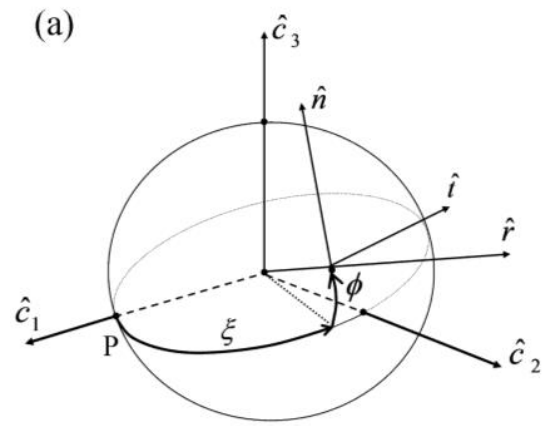

(b)

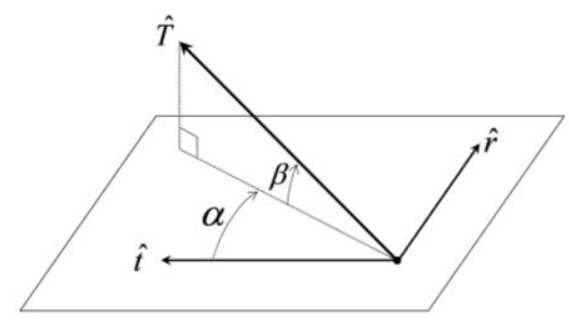

Fig. I: Reference frames (a) and thrust angles (b)

The problem at hand can be reformulated by using the dimensionless normalized time $\tau$,

$$
\tau:=t / t_{f} \quad \Rightarrow \quad \tau_{0} \equiv 0 \leq \tau \leq 1 \equiv \tau_{f}
$$

Let the dot denote the derivative with respect to $\tau$ hence forward. Equations [10] are rewritten as

$$
\dot{\boldsymbol{x}}=t_{f} \tilde{\boldsymbol{f}}\left(\boldsymbol{x}, \boldsymbol{u}, t_{f} \tau\right)=: \boldsymbol{f}(\boldsymbol{x}, \boldsymbol{u}, \boldsymbol{a}, \tau)
$$

where $\boldsymbol{a}$ collects all the unknown parameters of the problem ( $\boldsymbol{a}=t_{f}$ for the problem at hand).

As the space vehicle uses continuous thrust, minimizing the propellant consumption is equivalent to minimizing the time of flight $\left(t_{f}-t_{0}\right)$. Thus, as $t_{0}$ is set to 0 , the objective function is

$$
J=t_{f}
$$


II.II First-order necessary conditions for an extremal

With the intent of obtaining the necessary conditions for a local extremal, the Hamiltonian $H$ and the function of the boundary conditions $\tilde{\Phi}$ are introduced,

$$
\begin{aligned}
H(\boldsymbol{x}, \boldsymbol{u}, \boldsymbol{a}) & :=\lambda^{T} \boldsymbol{f}=\lambda_{1} t_{f} x_{4}+\lambda_{2} \frac{t_{f} x_{5}}{x_{1} \cos x_{3}}+\lambda_{3} \frac{t_{f} x_{6}}{x_{1}} \\
+ & \lambda_{4} t_{f}\left[-\frac{\mu}{x_{1}^{2}}+\frac{x_{5}^{2}+x_{6}^{2}}{x_{1}}+\frac{T}{\tilde{m}} \sin u_{1} \cos u_{2}\right] \\
+ & \lambda_{5} t_{f}\left[\frac{x_{5}}{x_{1}}\left(x_{6} \tan x_{3}-x_{4}\right)+\frac{T}{\tilde{m}} \cos u_{1} \cos u_{2}\right] \\
+ & \lambda_{6} t_{f}\left[\frac{x_{5}^{2}}{x_{1}} \tan x_{3}-\frac{x_{4} x_{6}}{x_{1}}+\frac{T}{\tilde{m}} \sin u_{2}\right] \\
\tilde{\Phi}\left(\boldsymbol{x}_{0}, \boldsymbol{x}_{f}, \boldsymbol{a}\right) & :=t_{f}+\boldsymbol{v}^{T} \boldsymbol{\psi}=t_{f}+v_{1}\left(x_{10}-R_{i}\right) \\
& +v_{2} x_{20}+v_{3} x_{30}+v_{4} x_{40}+v_{5}\left(x_{50}-\sqrt{\mu / R_{i}}\right) \\
& +v_{6} x_{60}+v_{7}\left(x_{1 f}-R_{f}\right)+v_{9} x_{3 f}+v_{9} x_{4 f} \\
& +v_{10}\left(x_{5 f}-\sqrt{\mu / R_{f}}\right)+v_{11} x_{6 f}
\end{aligned}
$$

where $x_{k 0}=x_{k}\left(t_{0}\right)$ and $x_{k f}=x_{k}\left(t_{f}\right)(k=1, \ldots, 6) ; \lambda$ and $v$ represent respectively the adjoint variable conjugate to the dynamics equations [15] and to the boundary conditions [13], with components $\left\{\lambda_{j}\right\}_{j=1, \ldots, 6}$ and $\left\{v_{j}\right\}_{j=1, \ldots, 11}$. The first-order necessary conditions for optimality include the adjoint (or costate) equations ${ }^{21}$, in conjunction with the related boundary conditons,

$$
\dot{\boldsymbol{\lambda}}=-\left[\frac{\partial H}{\partial \boldsymbol{x}}\right]^{T} \quad \lambda_{0}=-\left[\frac{\partial \tilde{\Phi}}{\partial \boldsymbol{x}_{0}}\right]^{T} \quad \lambda_{f}=\left[\frac{\partial \tilde{\Phi}}{\partial \boldsymbol{x}_{f}}\right]^{T}
$$

The optimal control $\boldsymbol{u}^{*}$ can be expressed as a function of the costates through the Pontryagin minimum principle,

$\boldsymbol{u}^{*}=\arg \min _{u}\left[\lambda_{4} \sin u_{1} \cos u_{2}+\lambda_{5} \cos u_{1} \cos u_{2}+\lambda_{6} \sin u_{2}\right]$

leading to

$$
\begin{gathered}
\beta^{*}=-\arcsin \frac{\lambda_{6}^{*}}{\sqrt{\lambda_{4}^{* 2}+\lambda_{5}^{* 2}+\lambda_{6}^{* 2}}} \\
\sin \alpha^{*}=-\frac{\lambda_{4}^{*}}{\sqrt{\lambda_{4}^{* 2}+\lambda_{5}^{* 2}}} \quad \cos \alpha^{*}=-\frac{\lambda_{5}^{*}}{\sqrt{\lambda_{4}^{* 2}+\lambda_{5}^{* 2}}}
\end{gathered}
$$

where the superscript "*" denotes the optimal value of the respective variable. Equations [21]-[22] imply the also the stationariety of $H$ with respect to $\boldsymbol{u}$. Lastly, the parameter condition ${ }^{21}$ must hold, and yields

$$
\int_{0}^{1}\left[\frac{\partial H}{\partial \boldsymbol{a}}\right]^{T} d \tau+\left[\frac{\partial \tilde{\Phi}}{\partial \boldsymbol{a}}\right]^{T}=0 \Rightarrow \int_{0}^{1} \lambda^{T} \frac{\partial \boldsymbol{f}}{\partial t_{f}} d \tau+1=0
$$

After introducing the variable $\boldsymbol{\mu}$, Eq. [23] is equivalent to $\dot{\boldsymbol{\mu}}=-\left[\frac{\partial H}{\partial \boldsymbol{a}}\right]^{T}$ with $\quad \boldsymbol{\mu}_{0}=0$ and $\boldsymbol{\mu}_{f}-\left[\frac{\partial \tilde{\Phi}}{\partial \boldsymbol{a}}\right]^{T}=0$ [24]

However, the parameter condition can be transformed into an inequality constraint, due to homogeneity of the costate equations, in conjunction with Eqs. [21]-[22], in which the control angles are expressed as the ratios of adjoint variables. In fact, due to Eqs. [21]-[22], homogeneity implies that if $\lambda$ is proportional to $\lambda^{*}$ ( $\lambda=k_{\lambda} \lambda^{*} ; k_{\lambda}$ denotes a positive constant), then the final conditions are fulfilled at the minimum final time $t_{f}^{*}$. In contrast, the parameter condition is violated, because the integral of Eq. [23] is

$$
\int_{0}^{1} \lambda^{T} \frac{\partial \boldsymbol{f}}{\partial t_{f}} d \tau=k_{\lambda} \int_{0}^{1} \lambda^{* T} \frac{\partial \boldsymbol{f}}{\partial t_{f}} d \tau=-k_{\lambda} \neq-1
$$

Therefore, if the proportionality condition holds, the optimal control $\boldsymbol{u}^{*}$ can be found without considering the parameter condition, which can be replaced by

$$
\int_{0}^{1} \lambda^{T} \frac{\partial f}{\partial t_{f}} d \tau<0
$$

In the formulation of the optimization problem the Moon is assumed spherical, as well as its gravitational field. As no further external force affects the spacecraft motion, the optimal transfer path can be assumed to lie entirely on the $\left(\hat{c}_{1}, \hat{c}_{2}\right)$-plane, and can be reasonably conjectured to outperform any hypothetical alternative three-dimensional trajectory. In fact, due to symmetry of the gravitational field, any out-of-plane thrust maneuver has the only effect of rotating the instantaneous velocity and would imply a useless waste of propellant. Therefore, the problem of determining the minimum-fuel path can be simplified by assuming that at any time the out-of-plane variables equal 0 , i.e.

$$
\begin{aligned}
& \phi=0 \text { and } v_{n}=0 \\
& \lambda_{3}^{*}=0 \text { and } \lambda_{6}^{*}=0 \quad \Rightarrow \quad \beta^{*}=0
\end{aligned}
$$

Only the state equations [4], [5], [7], and [8], the respective adjoint equations, and Eq. [22] are needed for the purpose of determining the optimal planar ascent path. The remaining adjoint equations, together with the related boundary conditions, are identically satisfied if Eqs. [27]-[28] hold. In addition, Eq. [5] is ignorable, because no final condition is prescribed for the right ascension $x_{2}$, and $x_{2}$ does not appear in the right-handside of any state equation. This circumstance implies also that $\lambda_{2}=0 \forall \tau$. In the end, the optimal ascent path optimization problem can be formulated as a two-point boundary-value problem in which the unknowns are the initial values of the adjoint variables $\lambda_{1}, \lambda_{4}$, and $\lambda_{5}$, as well as the time of flight $t_{f}$. 


\section{II.III Optimal transfer trajectory}

This subsection addresses the numerical determination of the minimum-time transfer path from the initial to the final low lunar orbit. To this end, the first-order conditions for optimality are used, in conjunction with a simple implementation of swarming algorithm. This is a heuristic optimization technique, based on the use of a population of individuals (or particles). Selection of the globally optimal parameters is the result of a number of iterations, in which the individuals share their information. This optimization approach is extremely intuitive and easy-to-implement. Nevertheless, in the scientific literature ${ }^{6,7,22-25}$ several papers prove that the use of this method is effective for solving trajectory optimization problems.

In this work the optimal control problem involves continuous time-dependent control variables and can be translated into a parameter optimization problem through the first-order necessary conditions for optimality, which allow expressing the control variables as functions of the adjoint variables conjugate to the dynamics equations. The parameter set includes $\left\{\lambda_{10}, \lambda_{40}, \lambda_{50}, t_{f}\right\}$. The boundary conditions are represented by the three equality constraints [12] for $r_{f}$, $v_{r f}$, and $v_{t f}$, accompanied by the inequality constraint [26]. Once the optimal parameter set has been determined, the (planar) state and costate equations can be integrated, using Eq. [22] to express the control angle $\alpha$ as a function of the adjoint variables.

For the problem at hand the PSO algorithm employs 100 particles and is run for 500 iterations. The problem is solved by employing a set of canonical units: the Moon radius represents the distance unit $(1 \mathrm{DU}=1738 \mathrm{~km})$, whereas the time unit is such that $\mu=1 \mathrm{DU}^{3} / \mathrm{TU}^{2}$ (i.e. $1 \mathrm{TU}=1034.8 \mathrm{sec}$ ). The search space is defined by the inequalities $-1 \leq \lambda_{k 0} \leq 1 \quad(k=1,3,4)$ and $10 \mathrm{TU} \leq t_{f} \leq 50 \mathrm{TU}$. It is worth remarking that the ignorability of the parameter condition allows defining arbitrarily the range in which the initial values of the adjoint variables are sought. The swarming algorithm is capable of obtaining the optimal (planar) ascent trajectory with great accuracy. In fact, the errors on the desired final conditions are $\left|r_{f}^{*}-R_{f}\right|=3.357 \cdot 10^{-11} \mathrm{~km},\left|v_{r f}^{*}\right|=6.258 \cdot 10^{-8} \mathrm{~km} / \mathrm{sec}$, and $\left|v_{t f}^{*}-\sqrt{\mu / R_{f}}\right|=1.033 \cdot 10^{-7} \mathrm{~km} / \mathrm{sec}$, whereas the minimum time turns out to be $t_{f}^{*}=10.60 \mathrm{hrs}$. Figures II through $\mathrm{V}$ portray the state components associated with the optimal ascent trajectory and the related optimal control time history.

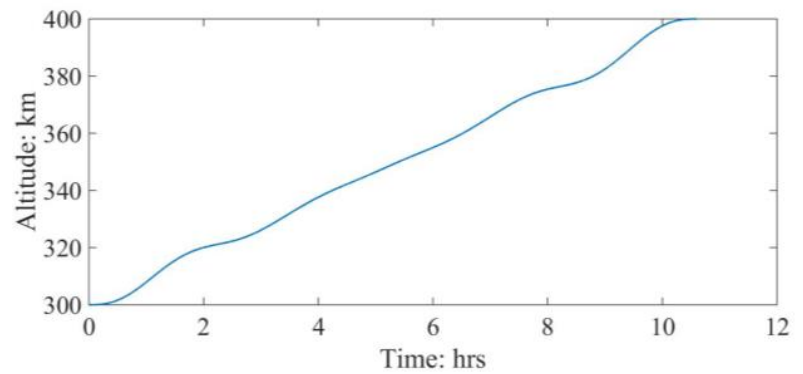

Fig. II: Optimal transfer path: altitude time history

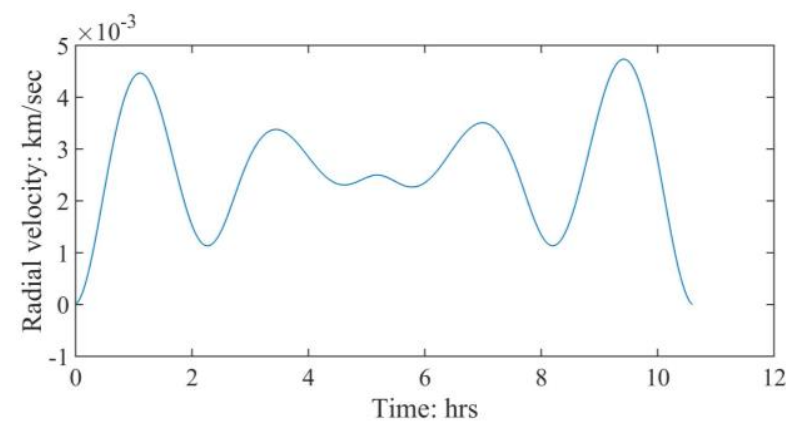

Fig. III: Optimal transfer path: radial velocity time history

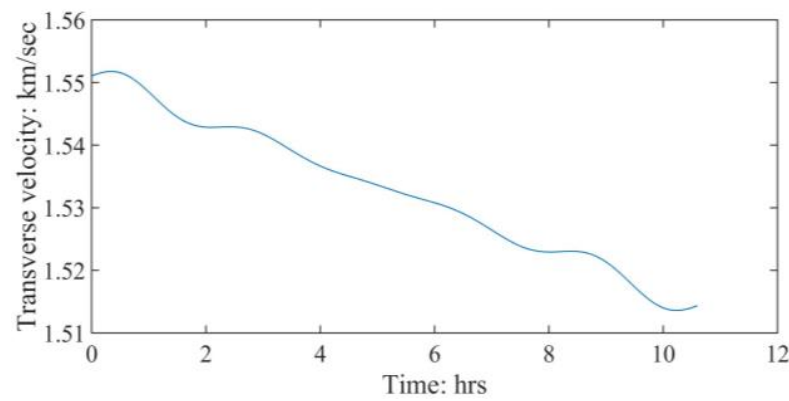

Fig. IV: Optimal transfer path: transverse velocity time history

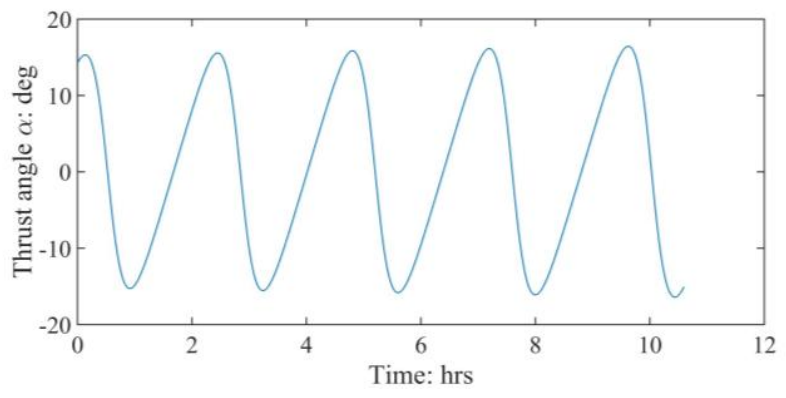

Fig. V: Optimal transfer path: thrust angle time history 
Evaluation of the matrices $H_{u u}$ and $\hat{\mathbf{S}}$ along the optimal path allows verifying that the second-order sufficient conditions for a minimum are both satisfied, and this represents the theoretical premise for a successful application of VTD-NOG.

\section{ORBIT PERTURBATIONS}

Due to the irregular mass concentrations, the lunar gravitational potential differs considerably from that generated by a spherical mass distribution. As a result, a relevant number of harmonics of the Moon potential are to be included in the dynamical simulations, in order to provide realistic results. Moreover, the Earth and Sun gravitational pull affects the spacecraft motion and can be modeled as a third body perturbation. This section is devoted to these two gravitational actions.

\section{III.I Harmonics of the lunar gravitational field}

Currently, some accurate models exist for the Moon gravitational fields, (a) Goddard gravity model 3 (GLGM3150) and (b) Lunar Prospector models, to name a few. This research employs the Lunar Prospector LPE100K model, which supplies the coefficients of zonal, tesseral, and sectorial harmonics of the Moon gravitational field up to order 100. These coefficients $\left(J_{l, m}\right.$ and $\left.\lambda_{l m}\right)$ appear in the classical equation of planetary gravitational potentials (per mass unit), written in terms of Legendre polynomials $P_{l m}$,

$$
\begin{aligned}
U & =\frac{\mu_{M}}{r}-\frac{\mu_{M}}{r} \sum_{l=2}^{\infty}\left(\frac{R_{M}}{r}\right)^{l} J_{l} P_{l 0}(\sin \phi) \\
& +\sum_{l=2}^{\infty} \sum_{m=1}^{l}\left(\frac{R_{M}}{r}\right)^{l} J_{l, m} P_{l m}(\sin \phi) \cos \left[m\left(\lambda_{g}-\lambda_{l m}\right)\right]
\end{aligned}
$$

where $\mu_{M}$ and $R_{M}$ are the Moon gravitational parameter and equatorial radius, $\phi$ and $\lambda_{g}$ are the satellite latitude and geographical longitude (taken from the Moon reference meridian ${ }^{26}$ ), whereas $r$ is its instantaneous radius. If $\lambda_{a}^{(\text {ref })}$ denotes the absolute longitude (taken counterclockwise from $\hat{c}_{1}$ ) of the Moon reference meridian, then the satellite absolute longitude is $\xi=\lambda_{a}^{(r e f)}+\lambda_{g}$.

In the $(\hat{r}, \hat{t}, \hat{n})$-frame, the gravitational acceleration is given by

$$
\boldsymbol{G}=\nabla U \quad \text { where } \nabla=\hat{r} \frac{\partial}{\partial r}+\frac{\hat{t}}{r \cos \phi} \frac{\partial}{\partial \lambda_{g}}+\frac{\hat{n}}{r} \frac{\partial}{\partial \phi}[30]
$$

The previous expression, together with Eq. [29], leads to obtaining the three components $\left(G_{r}, G_{t}, G_{n}\right)$ of the gravitational acceleration in the $(\hat{r}, \hat{t}, \hat{n})$-frame. Because $G_{r}$ includes the main gravitational term, the related disturbing accelerations are $a_{r}^{(H)}=G_{r}+\mu_{M} / r^{2}$, $a_{t}^{(H)}=G_{t}$, and $a_{n}^{(H)}=G_{n}$.

III.I Third body gravitational attraction

The Earth and Sun gravitational influence can be modeled as a third body perturbation. In general, the perturbing acceleration due to a third body can be expressed as

$$
\boldsymbol{a}_{3 B}=-\frac{\mu_{2}}{s_{3}^{3}\left(1+q_{3}\right)^{3 / 2}}\left[\boldsymbol{r}+\boldsymbol{s}_{3} q_{3} \frac{3+3 q_{3}+q_{3}^{2}}{1+\left(1+q_{3}\right)^{3 / 2}}\right]
$$

where $q_{3}:=\left(r^{2}-2 \boldsymbol{r} \cdot s_{3}\right) / s_{3}^{2}$. The symbol $\mu_{2}$ denotes the gravitational parameter of the third body, $\boldsymbol{s}_{3}$ represents its position vector relative to the main body (i.e., the Moon), and $s_{3}=\left|s_{3}\right|$. The previous expression makes use of the Battin-Giorgi ${ }^{27,28}$ approach to Encke's method for orbital perturbations. The perturbing acceleration $\boldsymbol{a}_{3 B}$ is to be projected into the $(\hat{r}, \hat{t}, \hat{n})$ frame, for its inclusion in the dynamics equations. To do this, $s_{3}$ needs to be projected into this frame.

As a first step, the Earth-centered inertial frame (ECI) and the Moon-centered inertial frame (MCI) are defined in relation to the heliocentric inertial frame (HCI). The latter reference system is associated with the unit vectors $\left(\hat{c}_{1}^{(S)}, \hat{c}_{2}^{(S)}, \hat{c}_{3}^{(S)}\right)$, where $\hat{c}_{1}^{(S)}$ is the vernal axis (corresponding to the intersection of the ecliptic plane with the Earth equatorial plane) and $\hat{c}_{3}^{(S)}$ points toward the Earth orbit angular momentum ${ }^{13}$. The ECIframe is associated with the unit vectors $\left(\hat{c}_{1}^{(E)}, \hat{c}_{2}^{(E)}, \hat{c}_{3}^{(E)}\right)$, where $\hat{c}_{1}^{(E)}$ is the vernal axis and $\hat{c}_{3}^{(E)}$

points toward the Earth rotation axis ${ }^{29}$. The ECI-frame and the HCI-frame are related through the ecliptic obliquity angle $\delta_{E}(=23.45 \mathrm{deg})$, [32]

$$
\left[\begin{array}{lll}
\hat{c}_{1}^{(E)} & \hat{c}_{2}^{(E)} & \hat{c}_{3}^{(E)}
\end{array}\right]^{T}=\mathbf{R}_{1}\left(-\delta_{E}\right)\left[\begin{array}{lll}
\hat{c}_{1}^{(S)} & \hat{c}_{2}^{(S)} & \hat{c}_{3}^{(S)}
\end{array}\right]^{T}
$$

According to Cassini's laws, the Moon's rotation axis $\hat{z}_{M}$ is coplanar with the Moon's orbit angular momentum $\boldsymbol{h}_{M}$ and the normal to the ecliptic plane $\hat{c}_{3}^{(S)}$. The two vectors $\hat{z}_{M}$ and $\boldsymbol{h}_{M}$ are located at opposite sides of the ecliptic pole $\hat{c}_{3}^{(S)}$, and both of them are subject to clockwise precession due to the Sun, with a period of 18.6 years. Hence, axis $\hat{c}_{3}$ of the MCI-frame can be properly identified as the rotation axis $\hat{z}_{M}$ at a reference epoch $t_{\text {ref }}, \hat{c}_{3}=\hat{z}_{M}\left(t_{\text {ref }}\right)$. If $\psi_{M}$ and $\delta_{M}$ denote respectively the precession angle and the Moon equator obliquity (separating $\hat{c}_{3}$ from $\hat{c}_{3}^{(S)}$ ), then 


$$
\left[\begin{array}{lll}
\hat{c}_{1} & \hat{c}_{2} & \hat{c}_{3}
\end{array}\right]^{T}=\mathbf{R}_{1}\left(\delta_{M}\right) \mathbf{R}_{3}\left(\psi_{M}^{(r e f)}\right)\left[\begin{array}{ccc}
\hat{c}_{1}^{(S)} & \hat{c}_{2}^{(S)} & \hat{c}_{3}^{(S)}
\end{array}\right]^{T}
$$

where $\psi_{M}^{(\text {ref })}(=-81.7 \mathrm{deg})$ represents the precession angle at $t_{\text {ref }}$ (set to 1 January 2020).

As a second step, the two-body-problem model is adopted to describe the Moon's motion around the Earth. If the Moon's orbit is approximated as circular, then its position vector relative to the Earth, denoted with $\boldsymbol{r}_{M}$, can be written in the ECI-frame in terms of $\Omega_{M}, i_{M}$, and $\theta_{M}$, i.e. the Moon RAAN, inclination, and (instantaneous) argument of latitude $\theta_{M}$,

$\boldsymbol{r}_{M}=r_{M}\left[\begin{array}{c}\cos \Omega_{M} \cos \theta_{M}-\sin \Omega_{M} \sin \theta_{M} \cos i_{M} \\ \sin \Omega_{M} \cos \theta_{M}+\cos \Omega_{M} \sin \theta_{M} \cos i_{M} \\ \sin \theta_{M} \sin i_{M}\end{array}\right]^{T}\left[\begin{array}{c}\hat{c}_{1}^{(E)} \\ \hat{c}_{2}^{(E)} \\ \hat{c}_{3}^{(E)}\end{array}\right]$

where $r_{M}$ is the instantaneous orbit radius (approximated to the constant value $384400 \mathrm{~km}$ ). The position vector of the Earth with respect to the Moon is simply $\boldsymbol{s}_{3}^{(E)}=-\boldsymbol{r}_{M}$. Due to precession of $\boldsymbol{h}_{M}$, the angles $\Omega_{M}$ and $i_{M}$ vary with a period of 18.6 years and can be assumed as constant only for relatively short times of flight. In order to project $\boldsymbol{s}_{3}^{(E)}$ along $(\hat{r}, \hat{t}, \hat{n})$, the relations between these three unit vectors and the MCIframe is needed,

$$
\left[\begin{array}{lll}
\hat{r} & \hat{t} & \hat{n}
\end{array}\right]^{T}=\mathbf{R}_{2}(-\phi) \mathbf{R}_{3}\left(\lambda_{a}\right)\left[\begin{array}{lll}
\hat{c}_{1} & \hat{c}_{2} & \hat{c}_{3}
\end{array}\right]^{T}
$$

Using Eqs. [32]-[35], the position vector $\boldsymbol{r}_{M}$ can be written in the desired frame,

$$
\begin{gathered}
\boldsymbol{r}_{M}=r_{M}\left[\begin{array}{c}
\cos \Omega_{M} \cos \theta_{M}-\sin \Omega_{M} \sin \theta_{M} \cos i_{M} \\
\sin \Omega_{M} \cos \theta_{M}+\cos \Omega_{M} \sin \theta_{M} \cos i_{M} \\
\sin \theta_{M} \sin i_{M}
\end{array}\right]^{T} \cdot \\
\cdot \mathbf{R}_{1}^{T}\left(\delta_{E}\right) \mathbf{R}_{3}^{T}\left(\psi_{M}^{(r e f)}\right) \mathbf{R}_{1}^{T}\left(\delta_{M}\right) \mathbf{R}_{3}^{T}\left(\lambda_{a}\right) \mathbf{R}_{2}(\phi)\left[\begin{array}{c}
\hat{r} \\
\hat{t} \\
\hat{n}
\end{array}\right]
\end{gathered}
$$

and the Earth perturbing acceleration, denoted with $\boldsymbol{a}_{3 B}^{(E)}=a_{r}^{(E)} \hat{r}+a_{t}^{(E)} \hat{t}+a_{n}^{(E)} \hat{n}$, can be calculated by means of Eq. [31], where $\boldsymbol{s}_{3}^{(E)}=-\boldsymbol{r}_{M}$.

Lastly, the two-body-problem model is adopted also to describe the Earth motion around the Sun. If the Earth orbit is approximated as circular, then its position vector, denoted with $\boldsymbol{r}_{E}$, can be written in the heliocentric inertial frame (HCI) in terms of $\theta_{E}$, i.e. the (instantaneous) Earth ecliptic longitude,

$$
\boldsymbol{r}_{E}=r_{E}\left[\begin{array}{lll}
\cos \theta_{E} & \sin \theta_{E} & 0
\end{array}\right]\left[\begin{array}{lll}
\hat{c}_{1}^{(S)} & \hat{c}_{2}^{(S)} & \hat{c}_{3}^{(S)}
\end{array}\right]^{T}
$$

where $r_{E}$ is the instantaneous orbit radius (approximated to the constant value of $1 \mathrm{AU}$ ). Using Eqs. [32], [33], [35], and [37], $\boldsymbol{r}_{E}$ can be rewritten as

$$
\begin{aligned}
& \boldsymbol{r}_{E}=r_{E}\left[\begin{array}{lll}
\cos \theta_{E} & \sin \theta_{E} & 0
\end{array}\right] \mathbf{R}_{3}^{T}\left(\psi_{M}^{(r e f)}\right) . \\
& \cdot \mathbf{R}_{1}^{T}\left(\delta_{M}\right) \mathbf{R}_{3}^{T}\left(\lambda_{a}\right) \mathbf{R}_{2}(\phi)\left[\begin{array}{lll}
\hat{r} & \hat{t} & \hat{n}
\end{array}\right]^{T}
\end{aligned}
$$

and this expression provides the projection along $(\hat{r}, \hat{t}, \hat{n})$. The position vector of the Sun with respect to the Moon is $\boldsymbol{s}_{3}^{(S)}=-\left(\boldsymbol{r}_{E}+\boldsymbol{r}_{M}\right)$, and can be written in the $(\hat{r}, \hat{t}, \hat{n})$-frame by means of Eqs. [36] and [38]. Finally, the Sun perturbing acceleration, denoted with $\boldsymbol{a}_{3 B}^{(S)}=a_{r}^{(S)} \hat{r}+a_{t}^{(S)} \hat{t}+a_{n}^{(S)} \hat{n}$, can be calculated through Eq. [31].

\section{VARIABLE-TIME-DOMAIN NEIGHBORING OPTIMAL GUIDANCE}

The Variable-Time-Domain Neighboring Optimal Guidance (VTD-NOG) uses the optimal trajectory as the reference path, with the final intent of determining the control correction at each sampling time $\left\{t_{k}\right\}_{k=0, \ldots, n_{S}}$, with $t_{0}=0$. These are the times at which the displacement between the actual trajectory, associated with $\boldsymbol{x}$, and the nominal trajectory, corresponding to $\boldsymbol{x}^{*}$, is evaluated, to yield

$$
d \boldsymbol{x}_{k} \equiv \delta \boldsymbol{x}_{k}=\boldsymbol{x}\left(t_{k}\right)-\boldsymbol{x}^{*}\left(t_{k}\right), \quad \text { with } t_{k}=\tau_{k} t_{f}^{(k)}
$$

where $t_{f}^{(k)}$ denotes the overall time of flight calculated at time $t_{k}$. The total number of sampling times, $n_{s}$, is unspecified, whereas the actual time interval between two successive sampling times is given and denoted with $\quad \Delta t_{S}, \Delta t_{S}=t_{k+1}-t_{k} \quad\left(k=0, \ldots, n_{S}-1\right)$. A fundamental ingredient needed to implement VTDNOG is the formula for determining $t_{f}^{(k)}$ at $t_{k}$.

IV.I Time-to-go updating law and termination criterion

The fundamental principle that underlies the VTDNOG scheme consists in finding the control correction $\delta \boldsymbol{u}(\tau)$ in the generic interval $\left[\tau_{k}, \tau_{k+1}\right]$ such that the second differential of $J$ is minimized ${ }^{13,15}$, while holding the first-order expansions of the state equations, the related final conditions, and the parameter condition Minimizing the second differential of $J$ is equivalent to solving the accessory optimization problem, defined in the interval $\left[\tau_{k}, 1\right]$. The solution of the same problem in the overall interval $[0,1]$ leads to deriving all the relations reported in Refs. 13 and 15. This means that the latter relations need to be extended to the generic 
interval $\left[\tau_{k}, 1\right]$. The analytical developments lead to deriving the feedback control correction,

$$
\delta \boldsymbol{u}=-H_{u u}^{-1}\left(H_{u x} \delta \boldsymbol{x}+H_{u a} d \boldsymbol{a}+H_{u \lambda} \delta \lambda\right)
$$

depending on the displacements (denoted with $\delta \boldsymbol{x}$, $\delta \lambda, d \boldsymbol{a}$ ) of the state, costate, and parameter vector from the respective optimal values. Equation [40] yields the control correction in each interval $\tau_{k} \leq \tau \leq \tau_{k+1}$.

Moreover, in Ref. 13, the following equation is derived:

$$
\left[\begin{array}{l}
d \boldsymbol{v} \\
d \boldsymbol{a}
\end{array}\right]=-\mathbf{V}_{k}^{-1} \mathbf{U}_{k}^{T} \delta \boldsymbol{x}_{k}-\mathbf{V}_{k}^{-1} \boldsymbol{\Theta} \delta \boldsymbol{\mu}_{k} \text { with } \boldsymbol{\Theta}:=\left[\begin{array}{c}
\mathbf{0}_{q x p} \\
\mathbf{I}_{p x p}
\end{array}\right]
$$

This relation differs from that included in the second order sufficient conditions, because $\delta \boldsymbol{\mu}_{k} \neq \mathbf{0}$ at the generic $\tau_{k}$ (unlike $\delta \boldsymbol{\mu}_{0}=\mathbf{0}$ ). The symbols $\boldsymbol{0}_{q \times p}$ and $\mathbf{I}_{p x p}$ denote respectively the null and the identity matrices (with dimensions indicated in the subscript), whereas matrices $\mathbf{U}_{k}$ and $\mathbf{V}_{k}$ are time-varying gain matrices defined in Ref. 13. The latter relation supplies the corrections $d \boldsymbol{v}$ and $d \boldsymbol{a}$ at $\tau_{k}$ as functions of $\delta \boldsymbol{x}_{k}$ (evaluated at $\tau_{k}$, cf. Eq. [39]), and $\delta \boldsymbol{\mu}_{k}$ (coming from the numerical integration of the respective linear differential equation Ref 13 in the preceding interval $\left.\left[\tau_{k-1}, \tau_{k}\right]\right)$. Actually, Eq. [41] contains the updating law of the total flight time $t_{f}$, which is included as a component of $\boldsymbol{a}$. Hence, if $d t_{f}^{(k)}$ denotes the correction on $t_{f}^{*}$ evaluated at $\tau_{k}$, then

$$
t_{f}^{(k)}=t_{f}^{*}+d t_{f}^{(k)}
$$

If the actual sampling interval $\Delta t_{S}$ is specified, the general formula for $\tau_{k}$ is

$$
\tau_{k+1}=\sum_{j=0}^{k} \frac{\Delta t_{S}}{t_{f}^{(j)}} \quad\left(k=1, \ldots, n_{S}-1\right)
$$

The overall number of intervals $n_{S}$ is found at the first occurrence of the following condition:

$$
\sum_{j=0}^{n_{S}} \frac{\Delta t_{S}}{t_{f}^{(j)}} \geq 1 \quad \Rightarrow \quad \tau_{n_{S}+1}=1
$$

It is worth stressing that the updating formula [42] derives directly from the natural extension of the accessory optimization problem to the time interval $\left[\tau_{k}, 1\right]$. In addition, the introduction of the normalized time $\tau$ now reveals its great utility. In fact, all the gain matrices are defined in the normalized interval $[0,1]$ and cannot become singular. Moreover, the limiting values $\left\{\tau_{k}\right\}_{k=1, \ldots, n_{S}-1}$ are calculated at each sampling time using

Eq. [43], while the sampling instants in the actual time domain are specified and equally-spaced. Also the termination criterion has a logical, consistent definition, and corresponds to the upper bound of the interval $[0,1]$, to which $\tau$ is constrained.

\section{IV.II Modified sweep method}

The definition of a neighboring optimal path requires the numerical backward integration of the sweep equations. A suitable integration technique is based on using the classical sweep equations in the interval $\left[\tau_{s w}, 1\right]$ (where $\tau_{s w}$ is sufficiently close to $\tau_{f}=1$ ) and then switching to $\hat{\mathbf{S}}$. However, due to Eq. [41], new relations are to be derived for $\hat{\mathbf{S}}$ and the related matrices.

As a preliminary step, the following relation is found for $\delta \lambda^{13}$ :

$$
\delta \boldsymbol{\lambda}=\left(\hat{\mathbf{S}}-\mathbf{W} \mathbf{m}^{T}\right) \delta \boldsymbol{x}-\mathbf{W n}^{T} d \boldsymbol{v}-\mathbf{W} \boldsymbol{\alpha} d \boldsymbol{a}
$$

where $\mathbf{W}:=\mathbf{U V}^{-1} \boldsymbol{\Theta}$. Further, considerable analytical developments ${ }^{13,15}$ (not reported for the sake of conciseness) lead to the following modified sweep equations:

$$
\begin{aligned}
\dot{\hat{\mathbf{S}}}= & -\hat{\mathbf{S}} \mathbf{A}+\hat{\mathbf{S}} \mathbf{B} \hat{\mathbf{S}}+\left[\hat{\mathbf{S}} \mathbf{D} \boldsymbol{\alpha}^{-1}+\mathbf{W F} \boldsymbol{\alpha}^{-1}+\mathbf{E} \boldsymbol{\alpha}^{-1}\right] \mathbf{m}^{T} \\
& -\mathbf{W} \mathbf{E}^{T}-\mathbf{W} \mathbf{D}^{T} \hat{\mathbf{S}}-\mathbf{C}-\mathbf{A}^{T} \hat{\mathbf{S}} \\
\dot{\mathbf{R}}^{T}= & \mathbf{R}^{T} \mathbf{B} \hat{\mathbf{S}}-\mathbf{R}^{T} \mathbf{A}-\mathbf{R}^{T} \mathbf{B W} \mathbf{m}^{T} \\
\dot{\mathbf{Q}}= & -\mathbf{R}^{T} \mathbf{B W} \mathbf{n}^{T} \\
\dot{\mathbf{n}}= & -\mathbf{R}^{T}(\mathbf{D}+\mathbf{B W} \boldsymbol{\alpha}) \\
\dot{\mathbf{m}}^{T}= & -\mathbf{m}^{T} \mathbf{A}+\mathbf{m}^{T} \mathbf{B} \hat{\mathbf{S}}-\mathbf{m}^{T} \mathbf{B W} \mathbf{m}^{T} \\
& -\mathbf{E}^{T}-\mathbf{D}^{T} \hat{\mathbf{S}}+\mathbf{D}^{T} \mathbf{W} \mathbf{m}^{T} \\
\dot{\boldsymbol{\alpha}}= & \mathbf{D}^{T} \mathbf{W} \boldsymbol{\alpha}-\mathbf{F}-\mathbf{m}^{T} \mathbf{B W} \boldsymbol{\alpha}-\mathbf{m}^{T} \mathbf{D}
\end{aligned}
$$

In the end, the gain matrices involved in the sweep method, i.e. $\mathbf{S}, \hat{\mathbf{S}}, \mathbf{R}, \mathbf{Q}, \mathbf{n}, \mathbf{m}$, and $\boldsymbol{\alpha}$, can be backward integrated in two steps:

(a) in the interval $\left[\tau_{s w}, 1\right]$ the equations of the classical sweep method ${ }^{13}$, with the respective boundary conditions are used,

(b) in the interval $\left[0, \tau_{s w}\right]$ Eqs. [46]-[51] are used. The matrices $\mathbf{R}, \mathbf{Q}, \mathbf{n}, \mathbf{m}$, and $\boldsymbol{\alpha}$ are continuous across the switching time $\tau_{s w}$, whereas $\hat{\mathbf{S}}$ is given by $\hat{\mathbf{S}}:=\mathbf{S}-\mathbf{U V}^{-1} \mathbf{U}^{T} ; \tau_{s w}$ is set to 0.99 .

\section{IV.III Preliminary offline computations}

The implementation of NOG requires several preliminary computations that can be completed offline and stored in the onboard computer.

First of all, the optimal trajectory is to be determined, together with the related state, costate, and control variables, which are assumed as the nominal ones. In the time domain $\tau$ these can be either available 
analytically or represented as sequences of equallyspaced values, e.g.

$$
\boldsymbol{u}_{i}^{*}=\boldsymbol{u}^{*}\left(\tau_{i}\right) \quad\left(i=0, \ldots, n_{D} ; \tau_{0}=0 \text { and } \tau_{n_{D}}=1\right)[52]
$$

However, in the presence of perturbations, NOG determines the control corrections $\delta \boldsymbol{u}(\tau)$ in each interval $\left[\tau_{k}, \tau_{k+1}\right]$, where the values $\left\{\tau_{k}\right\}$ never coincide with the equally-spaced values $\left\{\tau_{i}\right\}$ of Eq. [52]. Hence, regardless of the number of points used to represent the control correction $\delta \boldsymbol{u}(\tau)$ in $\left[\tau_{k}, \tau_{k+1}\right]$, it is apparent that a suitable interpolation is to be adopted for the control variable $\boldsymbol{u}^{*}$ (provided that no analytical expression is available). In this way, the value of $\boldsymbol{u}^{*}$ can be evaluated at any arbitrary time in the interval $0 \leq \tau \leq 1$. For the same reason also the nominal state $\boldsymbol{x}^{*}$ and costate $\lambda^{*}$ need to be interpolated. If a sufficiently large number of points is selected (e.g., $n_{D}=1001$ ), then piecewise linear interpolation is a suitable option. The successive step is the analytical derivation of the matrices

$$
\begin{aligned}
& \left\{f_{x}, f_{u}, f_{a}, H_{x x}, H_{x u}, H_{x \lambda}, H_{x a}, H_{u x}, H_{u u}, H_{u a}, H_{u \lambda},\right. \\
& H_{a x}, H_{a u}, H_{a a}, H_{a \lambda}, \psi_{x_{f}}, \psi_{x_{0}}, \psi_{a}, \tilde{\Phi}_{x_{0} x_{0}}, \tilde{\Phi}_{x_{0} a}, \tilde{\Phi}_{x_{f} x_{f}}, \\
& \left.\tilde{\Phi}_{x_{f} a}, \tilde{\Phi}_{a x_{f}}, \tilde{\Phi}_{a a}\right\}
\end{aligned}
$$

Then, they are evaluated along the nominal trajectory, and used to define also the matrices $\mathbf{A}, \mathbf{B}, \mathbf{C}$, $\mathbf{D}, \mathbf{E}$, and $\mathbf{F}$. Each element of these matrices, together with those of the matrices of Eq. [53], are linearly interpolated. Subsequently, the two-step backward integration of the sweep equations described in Section III.II is performed and yields the gain matrices $\hat{\mathbf{S}}, \mathbf{R}, \mathbf{m}$, $\mathbf{Q}, \mathbf{n}$, and $\boldsymbol{\alpha}$, using also the analytic expressions of $\mathbf{W}$, $\mathbf{U}$, and $\mathbf{V}$ (written in terms of $\mathbf{R}, \mathbf{m}, \mathbf{Q}, \mathbf{n}$, and $\boldsymbol{\alpha}$ ). The linear interpolation of all the matrices not yet interpolated concludes the preliminary computations.

\section{IV.IV VTD-NOG \& PD-RM algorithm structure}

On the basis of the optimal reference path, using the nominal quantities computed offline, at each time $\tau_{k}$ the VTD-NOG algorithm determines the time of flight and the control correction. Specifically, the following steps implement the feedback guidance scheme:

1. Set the actual sampling time interval $\Delta t_{S}$.

2. At each time $\tau_{k}\left(k=0, \ldots, n_{S}-1 ; \tau_{0}=0\right)$

a. Evaluate $\delta \boldsymbol{x}_{k}$ through Eq. [39];

b. Assume the value of $\delta \boldsymbol{\mu}$ calculated at the end of the previous interval $\left[\tau_{k-1}, \tau_{k}\right]$ as $\delta \boldsymbol{\mu}_{k}\left(\delta \boldsymbol{\mu}_{0}=\mathbf{0}\right)$;

c. Calculate the correction $d t_{f}^{(k)}$ and the updated time of flight $t_{f}^{(k)}$ by means of Eqs. [41]-[42]; d. Calculate the limiting value $\tau_{k+1}$ using Eq. [43];

e. Evaluate $\delta \lambda_{k}$ through Eq. [45];

f. Integrate numerically the linear differential system for $\delta \boldsymbol{x}(t), \delta \lambda(t)$, and $\delta \boldsymbol{\mu}(t)$;

g. Determine the control correction $\delta \boldsymbol{u}(\tau)$ in $\left[\tau_{k}, \tau_{k+1}\right]$ through Eq. [40].

3. If Eq. [44] holds, then VTD-NOG terminates, otherwise point 2 is repeated after increasing $k$ by 1 .

Figure VI portrays a block diagram that illustrates the sample-data feedback structure of the NOG algorithm, in which the control and flight time corrections definitely depend on the state displacement $\delta \boldsymbol{x}$ (evaluated at specified discrete times) through the timevarying gain matrices, which are computed offline and stored onboard. The attitude control loop (encircled by the dotted line) is being described in detail in the following.

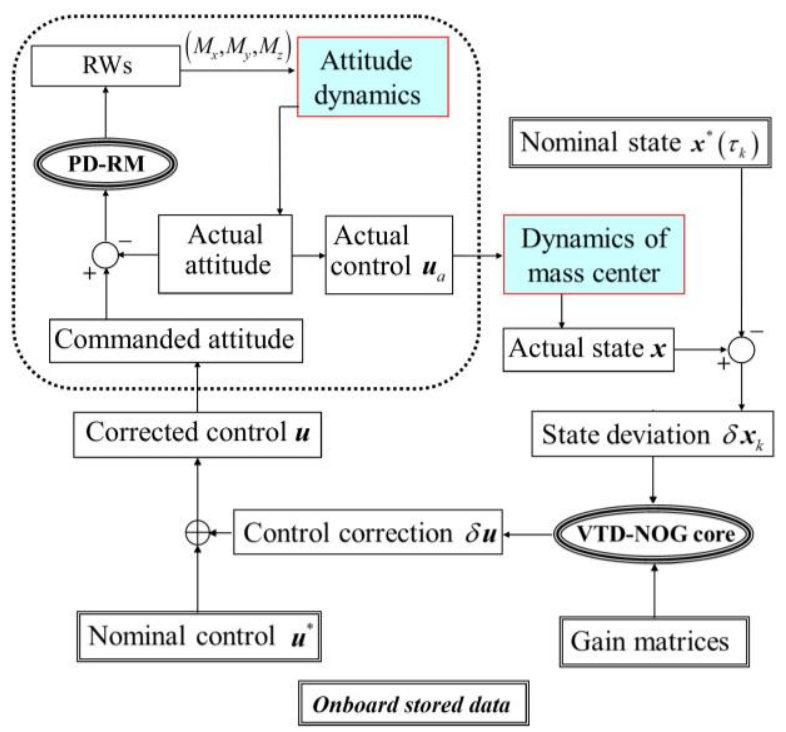

Fig. VI: Block diagram of VTD-NOG \& PD-RM

\section{PD ATTITUDE CONTROL USING ROTATION MATRICES}

The attitude control system is designed for the purpose of guaranteeing the correct spacecraft orientation, based on the corrected control $\boldsymbol{u}$ yielded by VTD-NOG. The output of the attitude control system is represented by the actual control $\boldsymbol{u}_{a}$ (cf. Fig. VI). The desired attitude is pursued using reaction wheels.

\section{V.I Commanded attitude}

With reference to Fig. VI, VTD-NOG yields the corrected control $\boldsymbol{u}$, i.e. the thrust direction identified by the angles $\alpha$ and $\beta$. Because the thrust is aligned with the longitudinal axis of the spacecraft, these two angles 
represent the commanded values, denoted with $\alpha_{c}$ and $\beta_{c}$, that the attitude control system must pursue.

The spacecraft instantaneous orientation is associated with the body frame $\left(\hat{x}_{b}, \hat{y}_{b}, \hat{z}_{b}\right)$, whose origin is in the instantaneous center of mass of the vehicle, its axes coincide with the principal axes of inertia, and $\hat{x}_{b}$ is aligned with the longitudinal axis. The two angles $\alpha_{c}$ and $\beta_{c}$ identifiy the desired orientation of $\hat{x}_{b}$, denoted with $\hat{x}_{b}^{(c)}$ and given by

$$
\begin{aligned}
\hat{x}_{b}^{(c)} & =\left[\begin{array}{c}
\cos \beta_{c} \sin \alpha_{c} \\
\cos \beta_{c} \cos \alpha_{c} \\
\sin \alpha_{c}
\end{array}\right]^{T}\left[\begin{array}{l}
\hat{r} \\
\hat{t} \\
\hat{n}
\end{array}\right] \\
& =\left[\begin{array}{c}
\cos \beta_{c} \sin \alpha_{c} \\
\cos \beta_{c} \cos \alpha_{c} \\
\sin \alpha_{c}
\end{array}\right]^{T} \mathbf{R}_{2}(-\phi) \mathbf{R}_{3}(\xi)\left[\begin{array}{l}
\hat{c}_{1} \\
\hat{c}_{2} \\
\hat{c}_{3}
\end{array}\right]
\end{aligned}
$$

The commanded unit vector $\hat{z}_{b}^{(c)}$ is chosen to be

$$
\hat{z}_{b}^{(c)}=\frac{\hat{c}_{3} \times \hat{x}_{b}^{(c)}}{\left|\hat{c}_{3} \times \hat{x}_{b}^{(c)}\right|}
$$

whereas $\hat{y}_{b}^{(c)}$ completes the right-hand sequence $\left(\hat{x}_{b}^{(c)}, \hat{y}_{b}^{(c)}, \hat{z}_{b}^{(c)}\right)$. It is apparent that in nominal flight conditions $\hat{z}_{b}^{(c)}$ lies in the equatorial plane and has positive component along the local nadir direction during the entire time of flight. Equations [54] and [55] lead to defining the commanded rotation matrix $\mathbf{R}_{c}$, which relates $\left(\hat{c}_{1}, \hat{c}_{2}, \hat{c}_{3}\right)$ to $\left(\hat{x}_{b}^{(c)}, \hat{y}_{b}^{(c)}, \hat{z}_{b}^{(c)}\right)$,

$$
\left[\begin{array}{lll}
\hat{x}_{b}^{(c)} & \hat{y}_{b}^{(c)} & \hat{z}_{b}^{(c)}
\end{array}\right]^{T}=\mathbf{R}_{c}\left[\begin{array}{lll}
\hat{c}_{1} & \hat{c}_{2} & \hat{c}_{3}
\end{array}\right]^{T}
$$

Matrix $\mathbf{R}_{c}$ represents the input that is supplied to the attitude control system.

\section{V.II Attitude dynamics}

A reaction wheel assembly is employed for the purpose of controlling the spacecraft attitude. Let $\boldsymbol{\omega}=\left[\begin{array}{lll}\omega_{x} & \omega_{y} & \omega_{z}\end{array}\right]^{T}$ be the body coordinates of the spacecraft angular velocity with respect to $\left(\hat{c}_{1}, \hat{c}_{2}, \hat{c}_{3}\right)$. The actual orientation is associated with the rotation matrix $\mathbf{R}$, which relates $\left(\hat{c}_{1}, \hat{c}_{2}, \hat{c}_{3}\right)$ to $\left(\hat{x}_{b}, \hat{y}_{b}, \hat{z}_{b}\right)$,

$$
\left[\begin{array}{lll}
\hat{x}_{b} & \hat{y}_{b} & \hat{z}_{b}
\end{array}\right]^{T}=\mathbf{R}\left[\begin{array}{lll}
\hat{c}_{1} & \hat{c}_{2} & \hat{c}_{3}
\end{array}\right]^{T}
$$

Then, the kinematics equations are given by ${ }^{30}$

$$
\dot{\mathbf{R}}=-\boldsymbol{\omega}^{\times} \mathbf{R} \quad \text { where } \quad \boldsymbol{\omega}^{\times}:=\left[\begin{array}{ccc}
0 & -\omega_{z} & \omega_{y} \\
\omega_{z} & 0 & -\omega_{x} \\
-\omega_{y} & \omega_{x} & 0
\end{array}\right]
$$

Moreover, the attitude dynamics equations are

$$
\mathbf{I} \dot{\boldsymbol{\omega}}+\boldsymbol{\omega}^{\times} \mathbf{I} \boldsymbol{\omega}=\boldsymbol{M}_{c}
$$

where $\mathbf{I}=\operatorname{diag}\left\{I_{x}, I_{y}, I_{z}\right\}$ is the spacecraft inertia matrix, whereas $\boldsymbol{M}_{c}=\left[\begin{array}{lll}M_{c x} & M_{c y} & M_{c z}\end{array}\right]^{T}$ are the body coordinates of the control torque generated by the reaction wheel assembly. Let $\overline{M_{c x}}, \overline{M_{c y}}, \overline{M_{c z}}$ denote the maximum torque components (about each body axis) that the reaction wheel assembly can generate. In order to take into account these limits, the variable $\tilde{\boldsymbol{M}}_{c}=\left[\begin{array}{lll}\tilde{M}_{c x} & \tilde{M}_{c y} & \tilde{M}_{c z}\end{array}\right]^{T}$ is introduced, related to $\boldsymbol{M}_{c}$ through the following equation:

$$
M_{c x}=\operatorname{sat}_{\overline{M_{c x}}}\left(\tilde{M}_{c x}\right)=\left\{\begin{array}{lll}
\overline{M_{c x}} & \text { if } & \tilde{M}_{c x}<-\overline{M_{c x}} \\
\tilde{M}_{c x} & \text { if } & -\overline{M_{c x}} \leq \tilde{M}_{c x} \leq \overline{M_{c x}}[60] \\
\overline{M_{c x}} & \text { if } & \tilde{M}_{c x}>\overline{M_{c x}}
\end{array}\right.
$$
and through similar equations for $M_{c y}$ and $M_{c z}$. Variable $\tilde{\boldsymbol{M}}_{c}$ represents the attitude control input for the spacecraft. Note that a model for the reaction wheel assembly is not included since it can be neglected for practical purposes ${ }^{31}$.

\section{V.III Attitude control law}

As the commanded attitude is specified by the rotation matrix $\mathbf{R}_{c}$ (cf. Section V.I), it is convenient to employ a control law that uses directly rotation matrix $\mathbf{R}_{c}$ for determining the appropriate torque that the reaction wheels must generate. In fact, this avoids singularities or ambiguities that the conversion of $\mathbf{R}_{c}$ into other attitude representations (such as Euler angles or quaternions) would introduce.

The following PD-like attitude control action is applied $^{32}$ :

$$
\tilde{\boldsymbol{M}}_{c}=-\mathbf{K}_{p} \sum_{i=1}^{3}\left(\boldsymbol{e}_{i} \times \mathbf{R}_{c} \mathbf{R}^{T} \boldsymbol{e}_{i}\right)-\mathbf{K}_{d} \boldsymbol{\omega}
$$

In the previous equations $\mathbf{K}_{p}=\operatorname{diag}\left\{k_{p x}, k_{p y}, k_{p z}\right\}$ and $\mathbf{K}_{d}=\operatorname{diag}\left\{k_{d x}, k_{d y}, k_{d z}\right\}$ are positive control gains, while $\left\{\boldsymbol{e}_{i}\right\}_{i=1,2,3}$ form the 3 by 3 identity matrix $\left[\begin{array}{lll}\boldsymbol{e}_{1} & \boldsymbol{e}_{2} & \boldsymbol{e}_{3}\end{array}\right]$. To analyze the convergence properties achieved by the considered controller, first it is worth noticing that quite often the commanded attitude $\mathbf{R}_{c}$ can be modeled as constant since it changes slowly with respect to the actual attitude $\mathbf{R}$. Proposition 2 of Ref. 32 implies that $\mathbf{R}$ converges to $\mathbf{R}_{c}$ locally and exponentially. In fact, Ref. 32 shows that after linearizing the closed-loop system composed of Eqs. [58] through [61] about $\mathbf{R}=\mathbf{R}_{c}$ and $\boldsymbol{\omega}=\mathbf{0}$, one obtains the following system: 


$$
\ddot{\boldsymbol{\zeta}}+\mathbf{I}^{-1} \mathbf{K}_{d} \dot{\boldsymbol{\zeta}}+2 \mathbf{I}^{-1} \mathbf{K}_{p} \boldsymbol{\zeta}=\mathbf{0}
$$

where $\zeta:=\left[\begin{array}{lll}\Phi & \Theta & \Psi\end{array}\right]^{T}$ and $\Psi, \Theta$, and $\Phi$ represent respectively the actual spacecraft 3-2-1 Euler angles relative to the commanded attitude. Then, it follows immediately that $\boldsymbol{\zeta} \rightarrow \mathbf{0}$.

\section{V.IV Gain selection}

The goal of the current subsection is presenting a method for determining at least first guess values for the gains $k_{p x}, k_{p y}, k_{p z}, k_{d x}, k_{d y}$, and $k_{d z}$. The method is here illustrated only for gains $k_{p x}$ and $k_{d x}$, because it can be extended easily to the other gains. Consider the first equation of the linearized closed-loop system in Eq. [62],

$$
\ddot{\Phi}+\frac{k_{d x}}{I_{x}} \dot{\Phi}+2 \frac{k_{p x}}{I_{x}} \Phi=0
$$

The corresponding characteristic equation in the Laplace domain is given by

$$
s^{2}+\frac{k_{d x}}{I_{x}} s+2 \frac{k_{p x}}{I_{x}}=0
$$

However, the value of $I_{x}$ varies during the flight. Let $\underline{I_{x}}$ and $\bar{I}_{x}$ be the minimum and maximum values of $I_{x}$. Then, the gains $k_{p x}$ and $k_{d x}$ are chosen so that for all $I_{x} \leq I_{x} \leq \overline{I_{x}}$ the solutions of Eq. [64] have damping ratio $\zeta_{x} \geq \underline{\zeta_{x}}$ and natural angular frequency $\omega_{n x} \geq \underline{\omega_{n x}}$. The lower bounds $\underline{\zeta_{x}}$ and $\underline{\omega_{n x}}$ are chosen based on experience and proceeding by trial-and-error. Because $2 k_{p x} / I_{x}=\omega_{n x}^{2}$ and $k_{d x} / I_{x}=2 \zeta_{x} \omega_{n x}$, it is easy to verify that the specifications $\zeta_{x} \geq \underline{\zeta_{x}}$ and $\omega_{n x} \geq \underline{\omega_{n x}}$ are fulfilled for all $I_{x} \leq I_{x} \leq \overline{I_{x}}$ by setting

$$
k_{p x}=\frac{\overline{\omega n x}_{n x}{ }^{2} \overline{I_{x}}}{2} \quad \text { and } \quad k_{d x}=2 \underline{\zeta_{x}} \underline{\omega_{n x}} \overline{I_{x}}
$$

\section{V.V Actual attitude}

The actual spacecraft orientation is defined by the instantaneous value of matrix $\mathbf{R}$, which identifies the actual orientation of axis $\hat{x}_{b}$ by means of Eq. [57]. Combination of the latter equation with Eq. [35] yields

$$
\left[\begin{array}{lll}
\hat{x}_{b} & \hat{y}_{b} & \hat{z}_{b}
\end{array}\right]^{T}=\mathbf{R R}_{3}^{T}(\xi) \mathbf{R}_{2}(\phi)\left[\begin{array}{lll}
\hat{r} & \hat{t} & \hat{n}
\end{array}\right]^{T}
$$

The actual thrust direction is aligned with $\hat{x}_{b}$, and can be written also as a function of the two actual thrust angles $\alpha_{a}$ and $\beta_{a}$ in the $(\hat{r}, \hat{t}, \hat{n})$-frame,

$$
\hat{T}_{a} \equiv \hat{x}_{b}=\left[\begin{array}{c}
\cos \beta_{a} \sin \alpha_{a} \\
\cos \beta_{a} \cos \alpha_{a} \\
\sin \beta_{a}
\end{array}\right]^{T}\left[\begin{array}{c}
\hat{r} \\
\hat{t} \\
\hat{n}
\end{array}\right]
$$

Comparison of Eqs. [66] and [67] leads to obtaining $\alpha_{a}$ and $\beta_{a}$ as functions of $\mathbf{R}, \phi$, and $\xi$.

\section{VTD-NOG \& PD-RM APPLIED TO \\ LOW-THRUST LUNAR TRANSFER}

The guidance and control methodology based on the joint use of VTD-NOG and PD-RM is applied to the low-thrust lunar transfer. The optimal transfer path is derived in a previous section and takes about 10.5 hours.

Further characteristics of the spacecraft are the initial mass $\tilde{m}_{0}=2400 \mathrm{~kg}$, the maximal torque generated by the reaction wheels about each body axis $\overline{M_{c x}}=\overline{M_{c y}}=\overline{M_{c z}}=0.5 \mathrm{~N} \mathrm{~m}$, and the time-varying inertia moments $I_{x}, I_{y}$, and $I_{z}$,

$$
I_{x}=I_{x 0}+\dot{I}_{x} t \quad I_{y}=I_{y 0}+\dot{I}_{y} t \quad I_{z}=I_{z 0}+\dot{I}_{z} t
$$

where

$$
I_{x 0}=1200 \mathrm{~kg} \mathrm{~m}^{2} \quad \dot{I}_{x}=-3.92 \cdot 10^{-4} \mathrm{~kg} \frac{\mathrm{m}^{2}}{\mathrm{sec}}
$$

$I_{y 0}=I_{y 0}=800 \mathrm{~kg} \mathrm{~m}^{2} \quad \dot{I}_{y}=\dot{I}_{z}=-2.61 \cdot 10^{-4} \mathrm{~kg} \frac{\mathrm{m}^{2}}{\mathrm{sec}}$

Moreover, the following values are selected for VTD-NOG \& PD-RM. The sampling interval $\Delta t_{s}$ is set to $1 \mathrm{~min}$, and the control gains are determined as follows. First, note that the constant thrust equals $T=n_{0} \tilde{m}_{0}$, whereas the maximum values for $I_{x}, I_{y}$, and $I_{z}$ are $\overline{I_{x}}=I_{x 0}, \overline{I_{y}}=I_{y 0}$, and $\overline{I_{z}}=I_{z 0}$. Proceeding by trial and error, the lower bounds for the natural frequencies and the damping coefficients are set to

$$
\begin{aligned}
& \underline{\omega_{n x}}=\underline{\omega_{n y}}=\underline{\omega_{n z}}=0.03 \mathrm{rad} \mathrm{sec}^{-1} \text { and } \\
& \underline{\zeta_{x}}=\underline{\zeta_{y}}=\underline{\zeta_{z}}=0.7
\end{aligned}
$$

Thus, by Eq. [65] and analogous equations for the other attitude control gains, one obtains $k_{p x}=0.54$, $k_{d x}=50.4, k_{p y}=k_{p y}=0.36, k_{d y}=k_{d z}=33.6$.

The first reason for the existence of deviations from nominal flight conditions is related to the fact that the commanded attitude does not coincide with the actual attitude. This is due to the fact that in general the commanded angles, yielded by VTD-NOG, are discontinuous across subsequent guidance intervals, while the actual thrust direction is constrained to vary with continuity, because it obeys the equations of attitude motion. This circumstance is pointed out also in Fig. VI, which illustrates clearly that the corrected 
control $\boldsymbol{u}$ does not coincide with the actual control $\boldsymbol{u}_{a}$, which affects the real dynamics of the center of mass.

Moreover, (modest) deviations from the nominal path occur also as a result of the gravitational perturbations due to Earth gravitational harmonics as well as to the Moon and Sun influence as third bodies. These perturbations were neglected while obtaining the nominal path. In the present context, these terms are to be considered, and include all the previously cited gravitational perturbations,

$$
a_{r / t / n}=a_{r / t / n}^{(H)}+a_{r / t / n}^{(E)}+a_{r / t / n}^{(S)}
$$

where superscripts $H, M$, and $S$ refer respectively to the lunar harmonics, the Earth, and the Sun contributions. With regard to the lunar gravitational field, all the harmonics with magnitude $\left|J_{l m}\right|>10^{-5}$ are included in the dynamical simulations, i.e. $J_{2} J_{6}, J_{7}, J_{9}, J_{22}$, and $J_{31}$.

As a first step, VTD-NOG \& PD-RM has been tested in order to evaluate these deviations, exclusively related to attitude motion and gravitational perturbations. The reference epoch at the initial time is set to 1 January 2020 at 12 UTC. The first column of Table I (denoted with GP, standing for "gravitational perturbation") reports the related results (obtained in a single simulation), i.e. the final displacements from the nominal final altitude, latitude, and velocity components, and testifies to the excellent accuracy of VTD-NOG \& PD-RM in this context.

However, further perturbations exist, related to the dynamical system itself. Monte Carlo (MC) campaigns are run, with the intent of obtaining some useful statistical information on the accuracy of the guidance and control algorithm of interest, in the presence of the existing perturbations, which are simulated stochastically. Usually, the thrust magnitude (and the related acceleration, as a result) exhibits small fluctuations. This time-varying behavior is modeled through a trigonometric series with random coefficients, $n_{0}^{p}=n_{0}\left[1+\sum_{k=1}^{5} \tilde{a}_{k} \sin \left(\frac{2 k \pi t}{t_{f}^{*}}\right)+\sum_{k=1}^{5} \tilde{a}_{k+5} \cos \left(\frac{2 k \pi t}{t_{f}^{*}}\right)\right]$

where $n_{0}^{p}$ denotes the perturbed value of $n_{0}$, whereas the coefficients $\left\{\tilde{a}_{k}\right\}_{k=1, \ldots, 10}$ have a random Gaussian distribution centered around the zero and a standard deviation equal to 0.01 . As the thrust magnitude is no longer constant, Eq. [1] is replaced by

$$
\frac{T}{\tilde{m}}=\frac{n_{0}^{p} \tilde{m}_{0}}{\tilde{m}} \quad \text { where } \quad \frac{\dot{\tilde{m}}}{\tilde{m}_{0}}=-\frac{n_{0}^{p}}{c}
$$

The symbol $\tilde{m}_{0}$ denotes the initial spacecraft mass. Moreover, errors on the initial attitude angles and rates are modeled. All of these displacements have Gaussian distribution and zero mean. Their standard deviation equals $10 \mathrm{deg}$ for the initial attitude (Euler) angles and $10 \mathrm{deg} / \mathrm{sec}$ for the initial angular velocity components (along the body axes).

At the end of VTD-NOG \& PD-RM, two statistical quantities are evaluated, i.e. the mean value and the standard deviation for all of the outputs of interest. The symbols $\overline{\Delta \chi}$ and $\chi^{(\sigma)}$ will denote the mean error (with respect to the nominal value) and standard deviation of $\chi$ henceforth. A single MC campaigns is performed, including all perturbations.

With reference to MC, Figs. VII through 16 portray the time histories of $n_{0}^{p}$, some relevant state variables, the torque components, as well as the principal rotation angle ${ }^{30}$ between the commanded and the actual rotation matrices $\mathbf{R}$ and $\mathbf{R}_{c}$. Moreover, Table I reports the statistics on the errors at injection and the time of flight. Inspection of this table reveals that VTD-NOG \& PD-RM guarantees orbit injection with very satisfactory accuracy, despite the relatively relaxed sampling time. Furthermore, the average time of flight is very close to the nominal value, and the corresponding standard deviation is modest.

As a final remark, the runtime of VTD-NOG \& PDRM on an Intel i5-3570K @ 3.40 GHz takes 13.37 min (while the nominal time of flight exceeds 10 hours), and this guarantees that the guidance and control algorithm at hand can be implemented in real time.

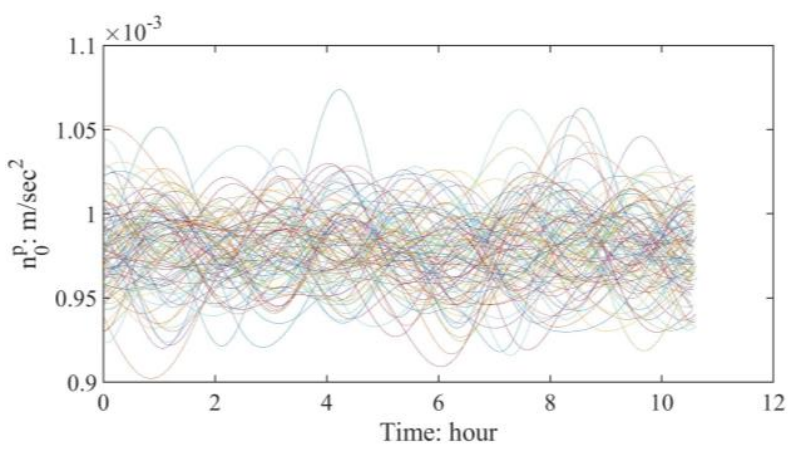

Fig. VII: Time histories of $n_{0}^{p}$ in the MC campaign

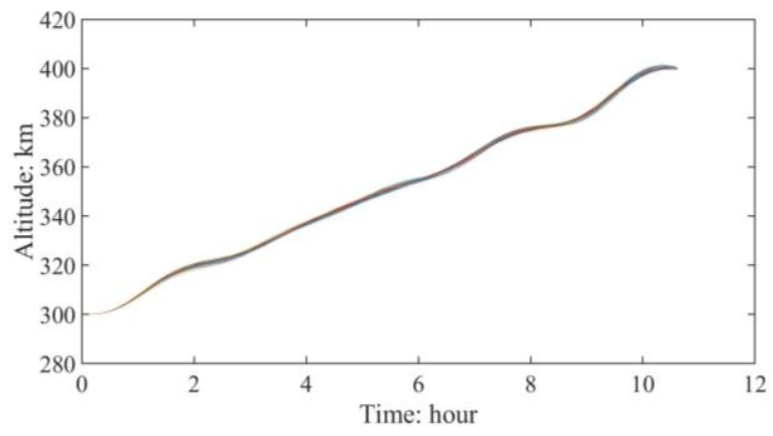

Fig. VIII: Altitude time histories obtained in the MC campaign 


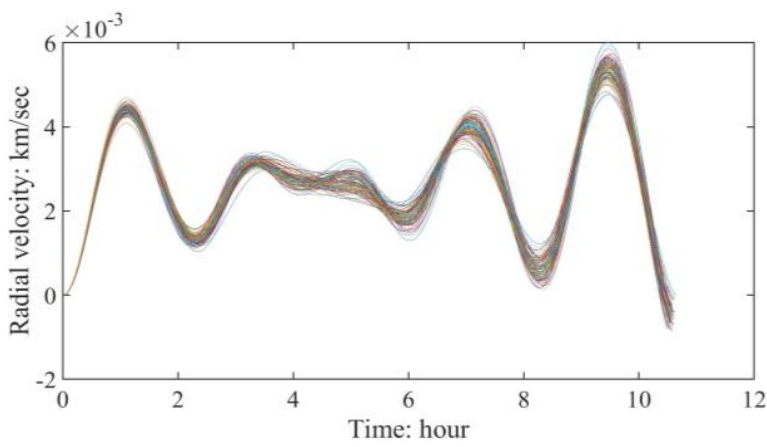

Fig. IX: Radial velocity time histories obtained in the MC campaign

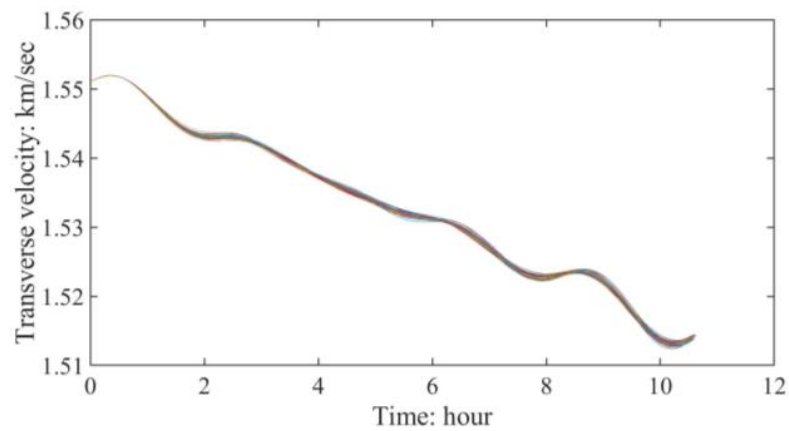

Fig. X: Transverse velocity time histories obtained in the MC campaign

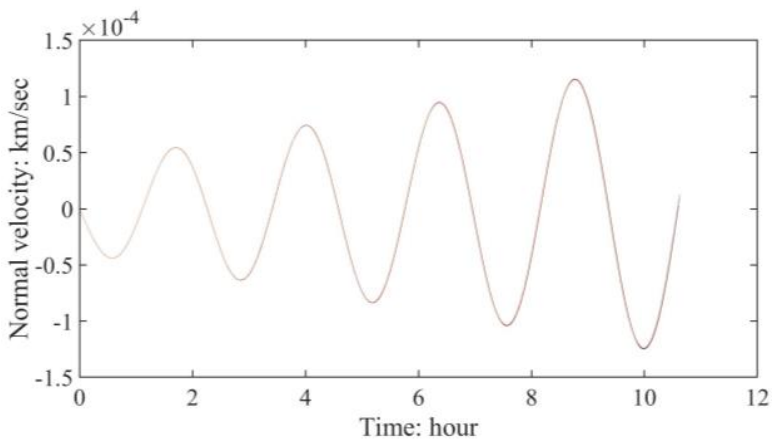

Fig. XI: Normal velocity time histories obtained in the MC campaign

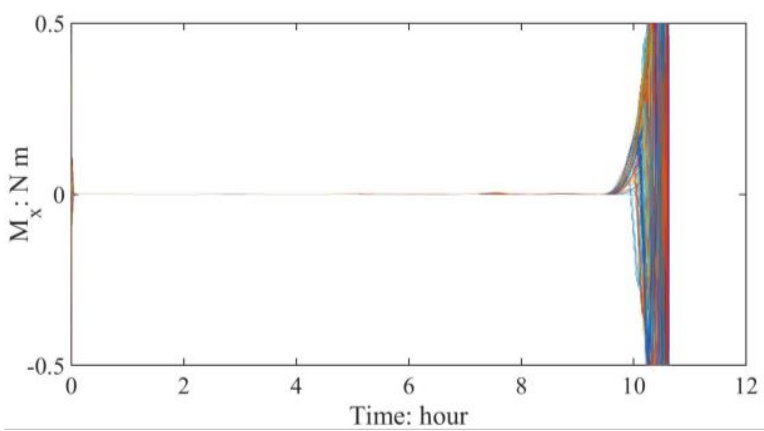

Fig. XII: Time histories of torque component $M_{x}$ obtained in the MC campaign

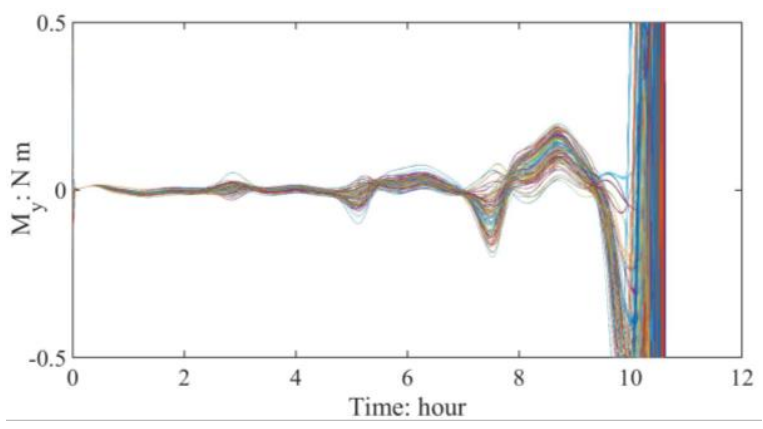

Fig. XIII: Time histories of torque component $M_{y}$ obtained in the MC campaign

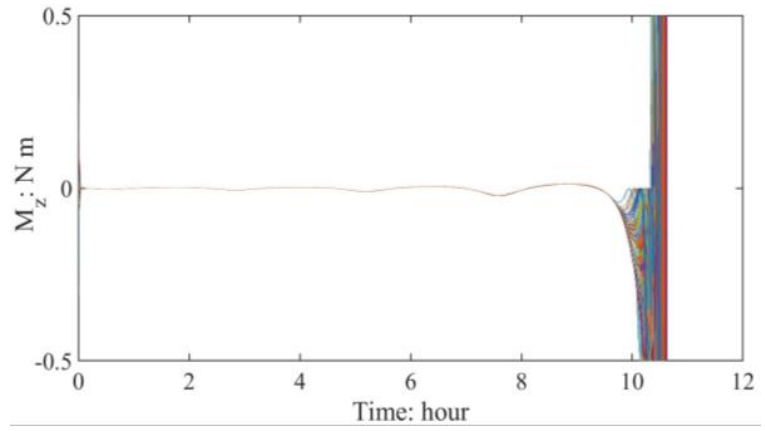

Fig. XIV: Time histories of torque component $M_{z}$ obtained in the MC campaign

\begin{tabular}{ccc}
\hline Statistics & GP & MC \\
\hline$\overline{\Delta r}_{f}(\mathrm{~km})$ & 0.31 & 0.33 \\
\hline$\overline{\Delta \phi}_{f}(\mathrm{deg})$ & $-5.9 \mathrm{e}-3$ & $-5.9 \mathrm{e}-3$ \\
\hline$\overline{\Delta v_{r f}}(\mathrm{~m} / \mathrm{sec})$ & -0.50 & -0.48 \\
\hline$\overline{\Delta v_{t f}}(\mathrm{~m} / \mathrm{sec})$ & -0.35 & -0.36 \\
\hline$\overline{\Delta v_{n f}}(\mathrm{~m} / \mathrm{sec})$ & $-8.9 \mathrm{e}-3$ & $-9.3 \mathrm{e}-3$ \\
\hline $\bar{t}_{f}(\mathrm{hrs})$ & 10.57 & 10.56 \\
\hline$r_{f}^{(\sigma)}(\mathrm{km})$ & $/$ & 0.33 \\
\hline$\phi_{f}^{(\sigma)}(\mathrm{deg})$ & $/$ & $3.8 \mathrm{e}-5$ \\
\hline$v_{r f}^{(\sigma)}(\mathrm{m} / \mathrm{sec})$ & $/$ & 0.18 \\
\hline$v_{t f}^{(\sigma)}(\mathrm{m} / \mathrm{sec})$ & $/$ & 2.28 \\
\hline$v_{n f}^{(\sigma)}(\mathrm{m} / \mathrm{sec})$ & $/$ & $9.9 \mathrm{e}-3$ \\
\hline$t_{f}^{(\sigma)}(\mathrm{hrs})$ & $/$ & $2.9 \mathrm{e}-2$ \\
\hline $\mathrm{Table} \mathrm{I:} \mathrm{Statistics}$
\end{tabular}

Table I: Statistics on the errors on the final state and on the time of flight 


\section{CONCLUDING REMARKS}

This work proposes VTD-NOG \& PD-RM, a new, general-purpose guidance and control algorithm for space vehicles, and describes its application to threedimensional lunar orbit transfers. VTD-NOG is a feedback guidance technique based upon minimizing the second differential of the objective function along the perturbed trajectory. This minimization principle leads to deriving all the corrective maneuvers. Due to adoption of a normalized time scale as the domain in which the nominal trajectory is defined, the gain matrices remain finite for the entire time of flight, while the updating law for the time of flight and the termination criterion find consistent definitions. VTDNOG identifies the trajectory corrections, and the commanded thrust angles. A proportional-derivative approach using rotation matrices (PD-RM) is employed in order to drive the actual spacecraft orientation toward the desired one. Reaction wheels are employed for attitude control and pursues this alignment condition. The new guidance and control architecture based on the joint use of VTD-NOG and PD-RM is applied to lowthrust lunar orbit transfer, with the intent of testing its capabilities. Oscillating perturbations of the propulsive thrust, gravitational perturbations, and errors on initial attitude are included in the dynamical model, and yield perturbed three-dimensional transfer paths. Monte Carlo simulations point out that orbit injection occurs with excellent accuracy, thus demonstrating that VTD-NOG \& PD-RM indeed represents an effective methodology for the application at hand. Due to its generality, VTDNOG \& PD-RM may be regarded as a promising approach for guidance and control of space vehicles employed in a wide variety of mission scenarios.

\section{REFERENCES}

${ }^{1}$ J. D. Betts, "Optimal Interplanetary Orbit Transfers by Direct Transcription," Journal of the Astronautical Sciences, Vol. 42, pp. 247-326, 1994

${ }_{2}^{2}$ P. J. Enright and B. A. Conway, "Optimal FiniteThrust Spacecraft Trajectories Using Collocation and Nonlinear Programming," Journal of Guidance, Control, and Dynamics, Vol. 14, pp. 981—985, 1991

3 A. Miele and T. Wang, "Multiple-Subarc Gradientrestoration Algorithm, Part 1: Algorithm Structure," Journal of Optimization Theory and Applications, Vol. 116, No. 1, pp. 1-17, 2003

${ }^{4}$ C. A. Kluever and B. L. Pierson, "Optimal LowThrust Earth-Moon Three-dimensional Trajectories," Journal of Guidance, Control, and Dynamics, Vol. 18, pp. 830-837, 1995

5 D. Hull, "Initial Lagrange Multipliers for the Shooting Method," Journal of Guidance, Control, and Dynamics Vol. 31, No. 5, pp. 1490-1492, 2008

${ }^{6}$ M. Pontani and B. A. Conway, "Optimal Low-Thrust Orbital Maneuvers via Indirect Swarming Method,"
Journal of Optimization Theory and Applications, Vol. 162, No. 1, pp. 272-292, 2014

${ }^{7}$ M. Pontani and B. A. Conway, "Minimum-Fuel Finite-Thrust Relative Orbit Maneuvers via Indirect Heuristic Method," Journal of Guidance, Control, and Dynamics, Vol. 38, No. 5, pp. 913-924, 2015

${ }^{8}$ H. H. Afshari, A. B. Novinzadeh, and J. Roshanian, "Determination of Nonlinear Optimal Feedback Law for Satellite Injection Problem Using Neighboring Optimal Control," American Journal of Applied Sciences, Vol. 6, No. 3, pp. 430-438, 2009

${ }^{9}$ H. Seywald and E. M. Cliff, "Neighboring Optimal Control Based Feedback Law for the Advanced Launch System," Journal of Guidance, Control, and Dynamics, Vol. 17, No. 3, pp. 1154-1162, 1994

${ }^{10}$ H. Yan, F. Fahroo, and I. M. Ross, "Real-Time Computation of Neighboring Optimal Control Laws," AIAA Guidance, Navigation and Control Conference and Exhibit, Monterey, CA, 2002; AIAA Paper 20024657

${ }^{11}$ S. N. Naidu, J. L. Hibey, and C. B. Charalambous, "Neighboring Optimal Guidance for Aeroassisted Orbital Transfer," IEEE Transactions on Aerospace and Electronic Systems, Vol. 29, No. 3, pp. 656663, 1993

${ }^{12}$ C. B. Charalambous, S. N. Naidu, and J. L. and Hibey, "Neighboring Optimal Trajectories for Aeroassisted Orbital Transfer Under Uncertainties," Journal of Guidance, Control, and Dynamics, Vol. 18, No. 3, pp. 478-485, 1995

13 M. Pontani, G. Cecchetti, and P. Teofilatto, "Variable-Time-Domain Neighboring Optimal Guidance, Part 1: Algorithm Structure," Journal of Optimization Theory and Applications, Vol. 166, pp. 76-92, 2015

${ }^{14}$ M. Pontani, G. Cecchetti, and P. Teofilatto, "Variable-Time-Domain Neighboring Optimal Guidance, Part 2: Application to Lunar Descent and Soft Landing," Journal of Optimization Theory and Applications, Vol. 166, pp. 93-114, 2015

15 M. Pontani, G. Cecchetti, and P. Teofilatto. "Variable-time-domain neighboring optimal guidance applied to space trajectories." Acta Astronautica, Vol. 115, pp. 102-120, 2015

${ }^{16}$ S. A. E. Berge, T. Olsson, G. Pionnier, M. Björk, C. Cbasset, T. Nordebäck, M. Rieschel, B. LübkeOssenbeck, and P. Zentgraf, "Advanced AOCS design on the first small GEO telecom satellite," Proceedings of the 60th International Astronautical Congress, Daejon, South Korea, 2009; paper IAC09.C1.6.10

17 A. Garulli, A. Giannitrapani, M. Leomanni, and F. Scortecci, "Autonomous low-earth-orbit stationkeeping with electric propulsion," Journal of Guidance, Control, and Dynamics, Vol. 34, No. 6, pp. 1683-1693, 2011 
${ }^{18}$ M. Pontani and F. Celani, "Neighboring optimal guidance and constrained attitude control applied to three-dimensional lunar ascent and orbit injection." Acta Astronautica, Vol. 156, pp. 78-91, 2019

${ }_{19}$ M. Pontani and F. Celani, "Variable-time-domain neighboring optimal guidance and attitude control for low-thrust orbit transfers," 2018 Space Flight Mechanics meeting, Orlando, FL, 2018; paper AIAA 2018-1456

${ }^{20} \mathrm{M}$. Pontani and F. Celani, "A new guidance and control architecture for accurate orbit injection," Advances in the Astronautical Sciences, Vol. 168, pp. 4133-4152, 1994; paper AAS 19-305

21 D. G. Hull, Optimal Control Theory for Applications, Springer International Edition, New York, NY, pp. 199-254, 2003

${ }^{22}$ M. Pontani and B. A. Conway, "Particle swarm optimization Applied to Space Trajectories," Journal of Guidance, Control, and Dynamics, Vol. 33, No. 5, pp. 1429-1441, 2010

${ }^{23}$ M. Pontani and B. A. Conway, "Particle swarm optimization applied to impulsive orbital transfers," Acta Astronautica, Vol. 74, pp. 141-155, 2012

${ }^{24}$ M. Pontani and B. A. Conway, "Optimal FiniteThrust Rendezvous Trajectories Found via Particle Swarm Algorithm," Journal of Spacecraft and Rockets, Vol. 50, pp. 1222-1234, 2013

${ }^{25}$ M. Pontani, "Particle Swarm Optimization of Ascent Trajectories of Multistage Launch Vehicles," Acta Astronautica, Vol. 94, No. 2, pp. 852-864, 2014

26 "A Standardized Lunar Coordinate System for the Lunar Reconnaissance Orbiter and Lunar Databases," LRO Project and LGCWG White Paper Version 5, Goddard Space Flight Center, Greenbelt, MD, 2008

${ }^{27}$ R. H. Battin, An Introduction to the Mathematics and Methods of Astrodynamics. AIAA Education Series, New York, NY, pp. 448—450, 490—494, 1987

${ }^{28}$ S. Giorgi, "Una Formulazione Caratteristica del Metodo di Encke in Vista dell'Applicazione Numerica." Scuola di Ingegneria Aerospaziale, Rome, Italy, 1964

29 J. E. Prussing and B. A. Conway, Orbital Mechanics, Oxford University Press, New York, NY, 2013, pp. $46-50$

${ }^{30}$ H. Schaub and J. L. Junkins, Analytical Mechanics of Space Systems, AIAA Education Series, Reston, VA, pp. 72-78, 95-97, 2003

31 M. J. Sidi, Spacecraft Dynamics and Control, Cambridge University Press, New York, 1997, p. 164

${ }^{32}$ N. A. Chaturvedi, A. K. Sanyal, and N. H. McClamroch, "Rigid-Body Attitude Control," IEEE Control Systems, Vol. 31, No. 3, pp. 30-51, 2011 